\title{
INTEGRATED DESIGN OF OBSERVER BASED FAULT DETECTION FOR A CLASS OF UNCERTAIN NONLINEAR SYSTEMS
}

\author{
Wei CHEN, Abdul Q. KHAN, Muhammmad ABID, Steven X. Ding \\ Institute of Automatic Control and Complex Systems \\ University of Duisburg-Essen, Bismarckstr. 81, 47057 Duisburg, Germany \\ e-mail: wei.chen.hai@stud.uni-due.de \\ \{aqkhan, mabid\}@pieas.edu.pk \\ steven.ding@uni-due.de
}

\begin{abstract}
Integrated design of observer based Fault Detection (FD) for a class of uncertain nonlinear systems with Lipschitz nonlinearities is studied. In the context of norm based residual evaluation, the residual generator and evaluator are designed together in an integrated form, and, based on it, a trade-off FD system is finally achieved in the sense that, for a given Fault Detection Rate (FDR), the False Alarm Rate (FAR) is minimized. A numerical example is given to illustrate the effectiveness of the proposed design method.
\end{abstract}

Keywords: fault detection, observers, nonlinear systems, optimization, robustness.

\section{Introduction}

The observer based fault detection and isolation technology is currently receiving much attention (Ding, 2008; Blanke et al., 2003; Chen and Patton, 1999; Patton et al., 2002). Generally speaking, an observer-based fault detection system consists of an observer-based residual generator and a residual evaluator. For linear systems, the observer-based FD technology has been well developed. Ding et al. (1993) as well as Qiu and Gertler (1993) proposed residual generator design schemes based on the $H_{\infty}$-optimization technique. Further on, the socalled $H_{-} / H_{\infty}$ design of residual generator, initiated by Ding et al. (1993) as well as Hou and Patton (1996), attracted lots of interest (Wang et al., 2007; Henry and Zolghadri, 2005). Recently, Ding et al. (2000a) and Zhang et al. (2005) proposed a unified solution which solves the $H_{i} / H_{\infty}$ (including $H_{-} / H_{\infty}$ and $H_{\infty} / H_{\infty}$ ) optimization problem, where $H_{i}$ represents all nonzero singular values of the transfer matrix from faults to the residual signal. For residual evaluation, there are two major strategies. One is statistic testing, which deals with the systems with stochastic behavior (Basseville and Nikiforov, 1993; Lai and Shan, 1999), and the other is norm based residual evaluation, which focuses on deterministic disturbance and model uncertainty (Frank and Ding, 1997).

In applications, an optimal trade-off between the
False Alarm Rate (FAR) and Fault Detection Rate (FDR) is of practical interest in designing an FD system. Ding et al. (2000b) extended the FAR and FDR concepts from the statistic context to characterize the performance of an FD system with norm based residual evaluation. As the FAR and FDR depend not only on the performance of the residual generator but also on the residual evaluator, an optimal trade-off between the FAR and FDR requires an integrated design of the residual generator and residual evaluator, which optimizes the performance of the whole FD system. This trade-off problem has been formulated in two ways: (1) given the FDR, minimize the FAR (Zhang and Ding, 2008), (2) given the FAR, maximize the FDR (Ding et al., 2000b).

On the other hand, since nonlinear systems are more common in practice, observer based FD techniques for nonlinear systems have also been studied extensively (Frank, 1994; Hammouri et al., 1999; Ferrari et al., 2007; Narasimhan et al., 2007; Shumsky, 2007; Edelmayer et al., 2004). There are many works dealing with Lipschitz nonlinear systems (Pertew et al., 2007; Chen and Saif, 2007; Rajamani and Ganguli, 2004; Yaz and Azemi, 1998; de Souza et al., 1993; Xie et al., 1996; Abbaszadeh and Marquez, 2008), since, under some conditions, more general nonlinear systems can be transformed into Lipschitz nonlinear systems (Rajamani, 1998). In this paper, we extend the integrated FD system design ap- 
proach which has been well developed for linear systems to uncertain Lipschitz nonlinear systems, based on the following formulation: Given the FDR, minimize the FAR.

The paper is organized as follows. After preliminaries addressed in Section 2, the problem of minimizing the FAR for a given FDR is formulated in Section 3. In Section 4, a solution to the integrated FD system design problem is proposed. Finally, in Section 5, the achieved results are illustrated with an example.

\section{Preliminaries}

Consider the following uncertain nonlinear systems:

$$
\Sigma_{\mathscr{S}}:\left\{\begin{array}{l}
\dot{x}=\bar{A} x+\phi(x, u)+\bar{B} u+\bar{E}_{d} d+E_{f} f, \\
y=\bar{C} x+\bar{D} u+\bar{F}_{d} d+F_{f} f
\end{array}\right.
$$

where $x \in \mathbb{R}^{n}$ is the state vector, $u \in \mathbb{R}^{m}$ is the control input, $y \in \mathbb{R}^{p}$ is the output vector, $f \in \mathbb{R}^{l}$ is the fault vector to be detected, and $d \in \mathbb{R}^{q}$ is the unknown input vector. Moreover, the matrices $\bar{A}, \bar{B}, \bar{E}_{d}, \bar{C}, \bar{D}, \bar{F}_{d}$ in (1) are uncertain of the form $\bar{X}=X+\Delta X$, where $X \in$ $\left\{A, B, E_{d}, C, D, F_{d}\right\}$ are known matrices with appropriate dimensions. Similarly, the matrices $E_{f}$ and $F_{f}$ are also known. $\Delta X \in\left\{\Delta A, \Delta B, \Delta E_{d}, \Delta C, \Delta D, \Delta F_{d}\right\}$ are norm bounded uncertainties and can be expressed as

$$
\left[\begin{array}{ccc}
\Delta A & \Delta B & \Delta E_{d} \\
\Delta C & \Delta D & \Delta F_{d}
\end{array}\right]=\left[\begin{array}{c}
E \\
F
\end{array}\right] \Delta(t)\left[\begin{array}{lll}
G & H & K
\end{array}\right],
$$

where $E, F, G, H, K$ are known matrices with appropriate dimensions and $\Delta(t)$ is bounded by

$$
\Delta(t)^{T} \Delta(t) \leq I
$$

The nonlinear function $\phi(x, u)$ is assumed to be Lipschitz in $x$ with a Lipschitz constant $\gamma$, i.e., $\forall x, \hat{x}, u$ :

$$
\|\phi(x, u)-\phi(\hat{x}, u)\| \leq \gamma\|x-\hat{x}\| .
$$

In addition, the following assumptions should be made throughout:

1. $A+\Delta A$ is asymptotically stable for all $\Delta A$.

2. $(C, A)$ is detectable.

The first assumption can be checked by the standard Lyapunov approach with LMI tools. The nonlinear observer based fault detection filter is designed as

$\Sigma_{\mathscr{F}}:\left\{\begin{array}{l}\dot{\hat{x}}=A \hat{x}+\phi(\hat{x}, u)+B u+L(y-C \hat{x}-D u), \\ r=y-C \hat{x}-D u,\end{array}\right.$

where $r \in \mathbb{R}^{p}$ is the residual signal and $L \in \mathbb{R}^{n \times p}$ is the observer gain. Denoting by $e=x-\hat{x}$ the estimation error, we have the following observer error dynamics:

$$
\Sigma_{\mathscr{E}}:\left\{\begin{aligned}
\dot{e}= & (A-L C) e+\Psi+(\Delta A-L \Delta C) x \\
& +(\Delta B-L \Delta D) u+\left(\bar{E}_{d}-L \bar{F}_{d}\right) d \\
& +\left(E_{f}-L F_{f}\right) f, \\
r= & C e+\Delta C x+\Delta D u+\bar{F}_{d} d+F_{f} f,
\end{aligned}\right.
$$

where

$$
\Psi=\phi(x, u)-\phi(\hat{x}, u) .
$$

Combining the system (1) and the error dynamics (2), the residual generator dynamics are as follows:

$$
\Sigma_{\mathscr{R}}:\left\{\begin{aligned}
\dot{x}_{0} & =\bar{A}_{0} x_{0}+\Psi_{0}+\bar{E}_{0} d_{0}+E_{0, f} f, \\
r & =\bar{C}_{0} x_{0}+\bar{F}_{0} d_{0}+F_{0, f} f,
\end{aligned}\right.
$$

where

$$
\begin{aligned}
& x_{0}=\left[\begin{array}{l}
x \\
e
\end{array}\right], \quad d_{0}=\left[\begin{array}{l}
u \\
d
\end{array}\right], \\
& {\left[\begin{array}{cc}
\bar{A}_{0} & \bar{E}_{0} \\
\bar{C}_{0} & \bar{F}_{0}
\end{array}\right]=\left[\begin{array}{cc}
A_{0} & E_{0} \\
C_{0} & F_{0}
\end{array}\right]+\left[\begin{array}{cc}
\Delta A_{0} & \Delta E_{0} \\
\Delta C_{0} & \Delta F_{0}
\end{array}\right],} \\
& A_{0}=\left[\begin{array}{cc}
A & 0 \\
0 & A-L C
\end{array}\right], \quad E_{0}=\left[\begin{array}{cc}
B & E_{d} \\
0 & E_{d}-L F_{d}
\end{array}\right], \\
& E_{0, f}=\left[\begin{array}{c}
E_{f} \\
E_{f}-L F_{f}
\end{array}\right], \quad \Psi_{0}=\left[\begin{array}{c}
\phi(x, u) \\
\phi(x, u)-\phi(\hat{x}, u)
\end{array}\right] \text {, } \\
& C_{0}=\left[\begin{array}{ll}
0 & C
\end{array}\right], \quad F_{0}=\left[\begin{array}{ll}
0 & F_{d}
\end{array}\right], \quad F_{0, f}=F_{f}, \\
& \Delta A_{0}=\left[\begin{array}{cc}
\Delta A & 0 \\
\Delta A-L \Delta C & 0
\end{array}\right], \\
& \Delta C_{0}=\left[\begin{array}{ll}
0 & \Delta C
\end{array}\right] \text {, } \\
& \Delta E_{0}=\left[\begin{array}{cc}
\Delta B & \Delta E_{d} \\
\Delta B-L \Delta D & \Delta E_{d}-L \Delta F_{d}
\end{array}\right], \\
& \Delta F_{0}=\left[\begin{array}{cc}
\Delta D & \Delta F_{d}
\end{array}\right], \\
& {\left[\begin{array}{cc}
\Delta A_{0} & \Delta E_{0} \\
\Delta C_{0} & \Delta F_{0}
\end{array}\right]=\left[\begin{array}{c}
\bar{E} \\
\bar{F}
\end{array}\right] \Delta(t)\left[\begin{array}{ll}
\bar{G} & \bar{H}
\end{array}\right]}
\end{aligned}
$$

where

$$
\begin{aligned}
& \bar{E}=\left[\begin{array}{ll}
E^{T} \quad(E-L F)^{T}
\end{array}\right]^{T}, \quad \bar{F}=F, \\
& \bar{G}=\left[\begin{array}{ll}
G & 0
\end{array}\right], \quad \bar{H}=\left[\begin{array}{ll}
H & K
\end{array}\right],
\end{aligned}
$$

and $d_{0}$ is assumed to be bounded by

$$
\left\|d_{0}\right\|_{2} \leq \delta_{d, \max }
$$

In the following, we will define the $H_{-}$index to measure the influence of the faults $f$ on the residual signal $r$ in (3). 
Definition 1. (Khan et al., 2009) Given the system $\Sigma_{\mathscr{R}}$ (3), assume that $d_{0}=0$. Then the $H_{-}$index can be defined as

$$
\left\|\Sigma_{\mathscr{R}}\right\|_{-}=\inf _{f \neq 0} \frac{\|r\|_{2}}{\|f\|_{2}} .
$$

For the $H_{-}$index to be larger than some positive number, $\beta$ can be defined as

$$
\left\|\Sigma_{\mathscr{R}}\right\|_{-}=\inf _{f \neq 0} \frac{\|r\|_{2}}{\|f\|_{2}} \geq \beta
$$

or

$$
\|r\|_{2} \geq \beta\|f\|_{2} .
$$

For the purpose of residual evaluation, the $\mathcal{L}_{2}$ norm of the residual signal is often adopted as the evaluation function and is also used in this paper,

$$
J=\|r\|_{2} \text {. }
$$

The decision logic of fault detection is as follows:

$$
\begin{aligned}
& J>J_{t h} \Longrightarrow \text { faulty } \\
& J \leq J_{t h} \Longrightarrow \text { fault }- \text { free }
\end{aligned}
$$

so that a false alarm is created if

$$
J>J_{t h} \text { for } f=0
$$

and a fault is detected if

$$
J>J_{t h} \text { for } f \neq 0 .
$$

Moreover, the following lemma is very useful.

Lemma 1. (Wang et al., 1992) Let $G, L, E, F(t)$ be real matrices of appropriate dimensions with $F(t)$ being a matrix function and $F(t)^{T} F(t) \leq I$. Then, for any $\epsilon>0$,

$$
L F(t) E+E^{T} F(t) L^{T} \leq \frac{1}{\epsilon} L L^{T}+\epsilon E^{T} E .
$$

\section{Problem formulation}

The objective of FD system design in this paper is to minimize the FAR under a given FDR. Ding (2008) defines the FDR in the norm based framework as

$$
\mathrm{FDR}=\frac{\beta \delta_{f, \min }}{J_{t h}}
$$

where $\beta$ is the $H_{-}$gain from faults to the residual signal with the assumption that there are no disturbances and uncertainties. In this case, the residual generator dynamics (3) become

$$
\left\{\begin{array}{l}
\dot{x}_{f}=(A-L C) x_{f}+\Psi+\left(E_{f}-L F_{f}\right) f, \\
r_{f}=C x_{f}+F_{f} f
\end{array}\right.
$$

so $\beta$ fulfills

$$
\left\|r_{f}\right\|_{2} \geq \beta\|f\|_{2}
$$

and $\delta_{f, \min }$ is the minimum size of the $f$ vector which is defined as a fault to be detected. We have

$$
\|f\|_{2} \geq \delta_{f, \min } .
$$

The physical meaning of this definition is that the larger the faults $\left(\delta_{f, \min }\right.$ is larger), the larger the FDR and the larger the threshold ( $J_{t h}$ is larger), the smaller the FDR.

Based on the definition of the FDR, when it is given $(\mathrm{FDR} \neq 0)$, the threshold should be set as

$$
J_{t h}=\beta \theta_{\mathrm{FDR}}
$$

where

$$
\theta_{\mathrm{FDR}}=\frac{\delta_{f, \min }}{\mathrm{FDR}} .
$$

In the work of Ding (2008), given a residual generator $r$ and $J_{t h}$, the set $\Omega_{F A}$ defined by

$$
\Omega_{\mathrm{FA}}=\left\{d \mid J>J_{t h} \quad \text { for } \quad f=0\right\}
$$

is called the set of disturbances that cause false alarms (SDFA). Since false alarms are created when $f=0$, in this case, the residual generator dynamics (3) become

$$
\left\{\begin{aligned}
\dot{x}_{0, d} & =\bar{A}_{0} x_{0, d}+\Psi_{0}+\bar{E}_{0} d_{0}, \\
r_{d} & =\bar{C}_{0} x_{0, d}+\bar{F}_{0} d_{0} .
\end{aligned}\right.
$$

So when the FDR is given and the threshold is set as (6), $\Omega_{\mathrm{FA}}$ can be expressed as

$$
\Omega_{\mathrm{FA}}=\left\{d_{0} \mid\left\|r_{d}\right\|_{2}>\beta \theta_{\mathrm{FDR}}\right\} .
$$

The size of $\Omega_{\mathrm{FA}}$ is a reasonable measurement of the rate of the false alarms. In this context, the size of $\Omega_{\mathrm{FA}}$ is interpreted as the FAR $(0 \leq \mathrm{FAR} \leq 1)$. Since the $\mathcal{L}_{2}$ norm of the disturbances considered is bounded by (4), when the FAR is small, only relative large disturbances will cause false alarms. So in the norm-based framework, the FAR can be expressed as

$$
\begin{gathered}
\left\|d_{0}\right\|_{2}>(1-\mathrm{FAR}) \delta_{d, \max } \\
\Longleftrightarrow\left\|r_{d}\right\|_{2}-\beta \theta_{\mathrm{FDR}}>0 .
\end{gathered}
$$

Note that, when FAR $=0$, since $\left\|d_{0}\right\|_{2}$ is bounded by (4), the condition $\left\|d_{0}\right\|_{2}>\delta_{d, \max }$ will never be fulfilled, which leads to the smallest size of $\Omega_{\mathrm{FA}}$. When FAR = 1 , the condition $\left\|d_{0}\right\|_{2}>0$ is almost always true, which leads to the largest size of $\Omega_{\mathrm{FA}}$.

Based on the relationship between the FDR and FAR which is represented in (8) and (9), our problem can be formulated as follows: Given the FDR, find an observer gain $L$ so that the FAR is minimized. 


\section{Solution to the integrated design problem}

Since the FDR is given, (9) can be transformed into

$$
\frac{\left\|r_{d}\right\|_{2}}{\beta \theta_{\mathrm{FDR}}}>1
$$

Then, based on (10), one sufficient condition for (8) is

$$
\left\|d_{0}\right\|_{2} \geq(1-\mathrm{FAR}) \delta_{d, \max } \frac{\left\|r_{d}\right\|_{2}}{\beta \theta_{\mathrm{FDR}}} .
$$

In (11), the FAR and FDR are connected, which is the key step for optimization. Since (11) is a sufficient condition, for the given FDR, the FAR calculated by (11) will be larger than or equal to its actual value. So a suboptimal solution can be achieved by minimizing the FAR based on (11). The FAR has been defined as

$$
0 \leq \mathrm{FAR} \leq 1
$$

In the following study, it is assumed that

$$
0 \leq \mathrm{FAR}<1
$$

Since the FAR should be minimized, this assumption will not lead to a conservative result. Based on it, (11) can be transformed into

$$
\left\|r_{d}\right\|_{2}-\beta \theta_{\mathrm{FDR}} \frac{\left\|d_{0}\right\|_{2}}{(1-\mathrm{FAR}) \delta_{d, \max }} \leq 0,
$$

where $\beta$ fulfills

$$
\left\|r_{f}\right\|_{2} \geq \beta\|f\|_{2}
$$

Based on (12) and (13), for a given FDR, the minimization of FAR can be solved in an iterative way as follows:

Step 1. Set the initial value of FAR.

Step 2. If there exist $\beta$ and an observer gain $L$ which fulfill (12) and (13), decrease (otherwise increase) the value of FAR till we get a minimum FAR.

The above algorithm gives an elegant tool for the minimization of the FAR provided the FDR is given. Now the question is how to check whether $\beta$ and $L$ exist in Step 2. To this end, the following theorem gives sufficient conditions for the existence of $\beta$ and $L$ based on the LMI technique.

Theorem 1. Given the residual generator dynamics (5) and (7), assume that $x_{f}(0)=0, x_{0, d}(0)=0$. Then

$$
\begin{aligned}
\left\|r_{d}\right\|_{2}-\beta \theta_{d f}\left\|d_{0}\right\|_{2} & \leq 0, \\
\left\|r_{f}\right\|_{2} & \geq \beta\|f\|_{2},
\end{aligned}
$$

where

$$
\theta_{d f}=\frac{\theta_{\mathrm{FDR}}}{(1-\mathrm{FAR}) \delta_{d, \max }}
$$

if there exist some $\epsilon>0, \beta>0, L$ and symmetric matrices $P_{1}>0, P_{2}>0, Q \leq 0$ so that

$$
\left[\begin{array}{cc}
\Omega_{1} & \Omega_{2} \\
* & \Omega_{3}
\end{array}\right] \leq 0
$$

$$
\left[\begin{array}{ccc}
N_{5} & Q\left(E_{f}-L F_{f}\right)+C^{T} F_{f} & \gamma Q \\
* & F_{f}^{T} F_{f}-\beta^{2} I & 0 \\
* & 0 & I
\end{array}\right] \geq 0
$$

where

$$
\begin{aligned}
& \Omega_{1}=\left[\begin{array}{cccc}
N_{1} & 0 & N_{3} & P_{1} E_{d}+\varepsilon G^{T} K \\
0 & N_{2} & 0 & P_{2}\left(E_{d}-L F_{d}\right) \\
* & * & N_{4} & \varepsilon H^{T} K \\
* & * & * & -\beta^{2} \theta_{d f}^{2} I+\varepsilon K^{T} K
\end{array}\right], \\
& \Omega_{2}=\left[\begin{array}{cccc}
0 & P_{1} E & 0 & \gamma P_{1} \\
C^{T} & P_{2}(E-L F) & \gamma P_{2} & 0 \\
0 & 0 & 0 & 0 \\
F_{d}^{T} & 0 & 0 & 0
\end{array}\right] \\
& \Omega_{3}=\left[\begin{array}{cccc}
-I & -F & 0 & 0 \\
* & -\varepsilon I & 0 & 0 \\
* & * & -I & 0 \\
* & * & * & -I
\end{array}\right] \\
& N_{1}=P_{1} A+A^{T} P_{1}+\varepsilon G^{T} G+I \text {, } \\
& N_{2}=P_{2}(A-L C)+(A-L C)^{T} P_{2}+I \text {, } \\
& N_{3}=P_{1} B+\varepsilon G^{T} H \text {, } \\
& N_{4}=-\beta^{2} \theta_{d f}^{2} I+\varepsilon H^{T} H, \\
& N_{5}=Q(A-L C)+(A-L C)^{T} Q-I+C^{T} C .
\end{aligned}
$$

Proof. The proof includes two parts.

Part 1: Let

$$
V_{d}=x_{0, d}^{T} P x_{0, d}, \quad P=\left[\begin{array}{cc}
P_{1} & 0 \\
0 & P_{2}
\end{array}\right]>0 .
$$

We have that

$$
\begin{aligned}
& r_{d}^{T} r_{d}-\beta^{2} \theta_{d f}^{2} d_{0}^{T} d_{0}+\dot{V}_{d} \leq 0 \\
& \quad \Longrightarrow \int_{0}^{\infty} r_{d}^{T} r_{d}-\beta^{2} \theta_{d f}^{2} \int_{0}^{\infty} d_{0}^{T} d_{0}+V_{d}(\infty) \leq 0 \\
& \quad \Longrightarrow\left\|r_{d}\right\|_{2}-\beta \theta_{d f}\left\|d_{0}\right\|_{2} \leq 0 .
\end{aligned}
$$

Thus (18) is a sufficient condition for (14).

We have

$$
\dot{V}_{d}=2 x_{0, d}^{T} P\left[\bar{A}_{0} x_{0, d}+\bar{E}_{0} d_{0}\right]+2 x_{0, d}^{T} P \Psi_{0} .
$$

Using the Cauchy-Schwarz inequality,

$$
2 x_{0, d}^{T} P \Psi_{0} \leq 2\left\|P x_{0, d}\right\|\left\|\Psi_{0}\right\|,
$$

and with the Lipschitz property of $\Psi_{0}$, we have

$$
\begin{aligned}
2\left\|P x_{0, d}\right\|\left\|\Psi_{0}\right\| & \leq 2 \gamma\left\|P x_{0, d}\right\|\left\|x_{0, d}\right\| \\
& \leq \gamma^{2} x_{0, d}^{T} P P x_{0, d}+x_{0, d}^{T} x_{0, d} .
\end{aligned}
$$


Substituting (20) and (21) into (19) we get

$$
\begin{aligned}
\dot{V}_{d} \leq & 2 x_{0, d}^{T} P\left[\bar{A}_{0} x_{0, d}+\bar{E}_{0} d_{0}\right] \\
& +\gamma^{2} x_{0, d}^{T} P P x_{0, d}+x_{0, d}^{T} x_{0, d} .
\end{aligned}
$$

Then a sufficient condition for (18) is

$$
\begin{aligned}
& \left(\bar{C}_{0} x_{0, d}+\bar{F}_{0} d_{0}\right)^{T}\left(\bar{C}_{0} x_{0, d}+\bar{F}_{0} d_{0}\right)-\beta^{2} \theta_{d f}^{2} d_{0}^{T} d_{0} \\
& \quad+2 x_{0, d}^{T} P\left[\bar{A}_{0} x_{0, d}+\bar{E}_{0} d_{0}\right]+\gamma^{2} x_{0, d}^{T} P P x_{0, d} \\
& +x_{0, d}^{T} x_{0, d} \leq 0 \\
& \Longleftrightarrow\left[\begin{array}{c}
x_{0, d} \\
d_{0}
\end{array}\right]^{T} \chi_{1}\left[\begin{array}{c}
x_{0, d} \\
d_{0}
\end{array}\right] \leq 0,
\end{aligned}
$$

where

$$
\chi_{1}=\left[\begin{array}{c}
\bar{C}_{0}^{T} \\
\bar{F}_{0}^{T}
\end{array}\right]\left[\begin{array}{ll}
\bar{C}_{0} & \bar{F}_{0}
\end{array}\right]+\left[\begin{array}{cc}
N_{6} & P \bar{E}_{0} \\
* & -\beta^{2} \theta_{d f}^{2} I
\end{array}\right]
$$

and

$$
N_{6}=P \bar{A}_{0}+\bar{A}_{0}^{T} P+\gamma^{2} P P+I
$$

so that

$$
\chi_{1} \leq 0 \Longrightarrow\left\|r_{d}\right\|_{2}-\beta \theta_{d f}\left\|d_{0}\right\|_{2} \leq 0 .
$$

Applying the Schur complement, we can rewrite (24) as

$$
\begin{aligned}
& {\left[\begin{array}{ccc}
N_{6} & P \bar{E}_{0} & \bar{C}_{0}^{T} \\
\bar{E}_{0}^{T} P & -\beta^{2} \theta_{d f}^{2} I & \bar{F}_{0}^{T} \\
\bar{C}_{0} & \bar{F}_{0} & -I
\end{array}\right] \leq 0} \\
& \Longleftrightarrow\left[\begin{array}{ccc}
P A_{0}+A_{0}^{T} P+\gamma^{2} P P+I & P E_{0} & C_{0}^{T} \\
E_{0}^{T} P & -\beta^{2} \theta_{d f}^{2} I & F_{0}^{T} \\
C_{0} & F_{0} & -I
\end{array}\right] \\
& +\left[\begin{array}{ccc}
P \Delta A_{0}+\Delta A_{0}^{T} P & P \Delta E_{0} & \Delta C_{0}^{T} \\
\Delta E_{0}^{T} P & 0 & \Delta F_{0}^{T} \\
\Delta C_{0} & \Delta F_{0} & 0
\end{array}\right] \leq 0 .
\end{aligned}
$$

Split the second matrix in the above inequality into

$$
\left[\begin{array}{ccc}
P \Delta A_{0}+\Delta A_{0}^{T} P & P \Delta E_{0} & \Delta C_{0}^{T} \\
\Delta E_{0}^{T} P & 0 & \Delta F_{0}^{T} \\
\Delta C_{0} & \Delta F_{0} & 0
\end{array}\right]=\chi_{2}+\chi_{2}^{T},
$$

where

$$
\chi_{2}=\left[\begin{array}{c}
P \bar{E} \\
0 \\
F
\end{array}\right] \Delta(t)\left[\begin{array}{lll}
\bar{G} & \bar{H} & 0
\end{array}\right] .
$$

Then, according to Lemma 1, 25) holds if there exists $\varepsilon>0$ so that

$$
\begin{gathered}
{\left[\begin{array}{ccc}
N_{7} & P E_{0} & C_{0}^{T} \\
E_{0}^{T} P & -\beta^{2} \theta_{d f}^{2} I & F_{0}^{T} \\
C_{0} & F_{0} & -I
\end{array}\right]+\frac{1}{\varepsilon}\left[\begin{array}{c}
P \bar{E} \\
0 \\
F
\end{array}\right]\left[\begin{array}{c}
P \bar{E} \\
0 \\
F
\end{array}\right]^{T}} \\
+\varepsilon\left[\begin{array}{lll}
\bar{G} & \bar{H} & 0
\end{array}\right]^{T}\left[\begin{array}{lll}
\bar{G} & \bar{H} & 0
\end{array}\right] \leq 0 .
\end{gathered}
$$

where

$$
N_{7}=P A_{0}+A_{0}^{T} P+\gamma^{2} P P+I .
$$

Applying the Schur complement yields

$$
\left[\begin{array}{cccc}
N_{8} & P E_{0}+\varepsilon \bar{G}^{T} \bar{H} & C_{0}^{T} & P \bar{E} \\
* & -\beta^{2} \theta_{d f}^{2} I+\varepsilon \bar{H}^{T} \bar{H} & F_{0}^{T} & 0 \\
* & * & -I & F \\
* & * & * & -\varepsilon I
\end{array}\right] \leq 0
$$

where

$$
N_{8}=P A_{0}+A_{0}^{T} P+\gamma^{2} P P+I+\varepsilon \bar{G}^{T} \bar{G} .
$$

Substituting

$$
P=\left[\begin{array}{cc}
P_{1} & 0 \\
0 & P_{2}
\end{array}\right]
$$

into the above inequality, we get

$$
\left[\begin{array}{cccccc}
N_{9} & 0 & N_{10} & N_{11} & 0 & P_{1} E \\
* & N_{12} & 0 & N_{13} & C^{T} & P_{2}(E-L F) \\
* & * & N_{14} & \varepsilon H^{T} K & 0 & 0 \\
* & * & * & N_{15} & F_{d}^{T} & 0 \\
* & * & * & * & -I & -F \\
* & * & * & * & * & -\varepsilon I
\end{array}\right] \leq 0
$$

where

$$
\begin{aligned}
N_{9} & =P_{1} A+A^{T} P_{1}+\varepsilon G^{T} G+\gamma^{2} P_{1} P_{1}+I, \\
N_{10} & =P_{1} B+\varepsilon G^{T} H, N_{11}=P_{1} E_{d}+\varepsilon G^{T} K, \\
N_{12} & =P_{2}(A-L C)+(A-L C)^{T} P_{2}+\gamma^{2} P_{2} P_{2}+I, \\
N_{13} & =P_{2}\left(E_{d}-L F_{d}\right), N_{14}=-\beta^{2} \theta_{d f}^{2} I+\varepsilon H^{T} H, \\
N_{15} & =-\beta^{2} \theta_{d f}^{2} I+\varepsilon K^{T} K .
\end{aligned}
$$

Finally, applying the Schur complement again, we get (16) of Theorem 1, which is a sufficient condition for (14).

Part 2: Let

$$
V_{f}(x)=x_{f}^{T} Q x_{f}, \quad Q \leq 0 .
$$

It holds that

$$
\begin{aligned}
& r_{f}^{T} r_{f}-\beta^{2} f^{T} f+\dot{V}_{f} \geq 0 \\
& \quad \Longrightarrow \int_{0}^{\infty} r_{f}^{T} r_{f}-\beta^{2} \int_{0}^{\infty} f^{T} f+V_{f}(\infty) \geq 0 \\
& \quad \Longrightarrow\left\|r_{f}\right\|_{2} \geq \beta\|f\|_{2},
\end{aligned}
$$

so (26) is a sufficient condition for (15). We have

$$
\begin{aligned}
\dot{V}_{f}= & 2 x_{f}^{T} Q\left[(A-L C) x_{f}+\left(E_{f}-L F_{f}\right) f\right] \\
& +2 x_{f}^{T} Q \Psi .
\end{aligned}
$$

Using the Cauchy-Schwarz inequality,

$$
2 x_{f}^{T} Q \Psi \geq-2\left\|Q x_{f}\right\|\|\Psi\|,
$$


and with the Lipschitz property of $\Psi$, we have

$$
\begin{aligned}
2\left\|Q x_{f}\right\|\|\Psi\| & \leq 2 \gamma\left\|Q x_{f}\right\|\left\|x_{f}\right\| \\
& \leq \gamma^{2} x_{f}^{T} Q Q x_{f}+x_{f}^{T} x_{f} .
\end{aligned}
$$

Substituting (28) and 29) into 27), we get

$$
\begin{aligned}
\dot{V}_{f} \geq & 2 x_{f}^{T} Q\left[(A-L C) x_{f}+\left(E_{f}-L F_{f}\right) f\right] \\
& -\gamma^{2} x_{f}^{T} Q Q x_{f}-x_{f}^{T} x_{f} .
\end{aligned}
$$

Then a sufficient condition for (26) is

$$
\begin{aligned}
& \left(C x_{f}+F_{f} f\right)^{T}\left(C x_{f}+F_{f} f\right)-\beta^{2} f^{T} f \\
& \quad+2 x_{f}^{T} Q(A-L C) x_{f}+2 x_{f}^{T} Q\left(E_{f}-L F_{f}\right) f \\
& \quad-\gamma^{2} x_{f}^{T} Q Q x_{f}-x_{f}^{T} x_{f} \geq 0 \\
& \Longleftrightarrow\left[\begin{array}{c}
x_{f} \\
f
\end{array}\right]^{T}\left[\begin{array}{cc}
N_{16} & N_{17} \\
* & F_{f}^{T} F_{f}-\beta^{2} I
\end{array}\right]\left[\begin{array}{c}
x_{f} \\
f
\end{array}\right] \geq 0,
\end{aligned}
$$

where

$$
\begin{aligned}
N_{16}= & Q(A-L C)+(A-L C)^{T} Q-\gamma^{2} Q Q \\
& -I+C^{T} C, \\
N_{17}= & Q\left(E_{f}-L F_{f}\right)+C^{T} F_{f} .
\end{aligned}
$$

Applying the Schur complement yields (17) of Theorem 1, which is a sufficient condition for (15). This completes the proof.

In Theorem 1, 16 and (17) are NMIs which can be approached by an advanced nonlinear optimization technique. A conservative solution could be achieved by setting

$$
Q=-P_{2}, \quad Y=P_{2} L .
$$

Then (16) and 17) are transformed into standard LMIs.

\section{Example}

In this section, an example is given to illustrate the achieved results.

Consider the FD problem of a system in the form of (1) with coefficient matrices:

$$
\begin{array}{rlr}
A & =\left[\begin{array}{ccc}
-6.5 & 3.9 & 5.2 \\
0 & -9.1 & 3.9 \\
1.3 & 3.9 & -7.8
\end{array}\right], \quad B=\left[\begin{array}{c}
1 \\
2 \\
1.5
\end{array}\right], \\
C & =\left[\begin{array}{ccc}
1 & 2 & -1 \\
2 & -1 & 3
\end{array}\right], & D=\left[\begin{array}{l}
0.5 \\
0.3
\end{array}\right], \\
E d & =\left[\begin{array}{ccc}
-0.3 & 1 & 0.6 \\
0 & 0.3 & 0.5 \\
0.4 & 0 & -0.2
\end{array}\right], & \\
E f & =\left[\begin{array}{cc}
1.3 & 0.65 \\
-0.39 & 1.04 \\
0.78 & -1.17
\end{array}\right], & \\
F d & =\left[\begin{array}{ccc}
0.7 & 1 & -0.3 \\
0 & 0.6 & 0.2
\end{array}\right],
\end{array}
$$

$$
\begin{aligned}
F f & =\left[\begin{array}{cc}
1.6 & 0 \\
0 & -1.6
\end{array}\right], \\
E & =\left[\begin{array}{c}
0.2 \\
0.3 \\
0.15
\end{array}\right], \\
\phi(x, u) & =0.5\left[\begin{array}{c}
\sin \left(x_{1}\right) \\
\cos \left(x_{2}\right) \\
0
\end{array}\right] \\
G & =\left[\begin{array}{ccc}
0.25 & 0.1 & 0.33
\end{array}\right], \quad H=0.12, \\
K & =\left[\begin{array}{lll}
0.16 & 0.23 & 0.31
\end{array}\right] .
\end{aligned}
$$

The faults which are defined to be detected are bounded by

$$
\|f\|_{2} \geq \delta_{f, \min }=5
$$

and the disturbances are bounded by

$$
\left\|d_{0}\right\|_{2} \leq \delta_{d, \max }=6 .
$$

Set FDR $=1$. Then, following the design procedure in Section 4 to minimize FAR, we get the optimal observer gain matrix as

$$
L_{\mathrm{opt}}=\left[\begin{array}{cc}
0.8175 & -0.3244 \\
-0.1393 & -0.4087 \\
0.3407 & 0.5156
\end{array}\right] \text {. }
$$

The corresponding $H_{-}$gain $\beta$ in $(12)$ is $\beta=1.5038$, so, according to (6), the threshold is set as

$$
J_{t h}=\beta \frac{\delta_{f, \min }}{\mathrm{FDR}}=7.5191 .
$$

In the simulation study, the simulation time is set to be 3000 seconds and the control input is a step signal $u=0.4$ (step time at 0 ). The unknown disturbances are, respectively, a sine wave $0.14 \sin (10 t)$, a step signal (step time at 0 ) of amplitude 0.25 , and a continuous signal taking value randomly from a uniform distribution between $[-0.2,0.2]$. The residual signal is evaluated in a time window of 10 seconds $J(t)=\left(\int_{t-10}^{t} r^{T} r \mathrm{~d} t\right)^{\frac{1}{2}}$. Fault 1 appears at the 1750-th second as a step function $f_{1}=0.5$. Fault 2 appears at the 1950-th second as a step function $f_{2}=-0.4$. The simulation results are shown in Fig. 1 . We can see that, when there are no faults, $J$ is always less than the threshold, and hence no false alarm is created. When faults appear, $J$ is turned to be greater than the threshold, which means that the faults are successfully detected. This demonstrates the achieved results.

\section{Conclusions}

In this paper, an integrated design of observer based fault detection for a class of uncertain nonlinear systems has been developed, which is a trade-off design between the 


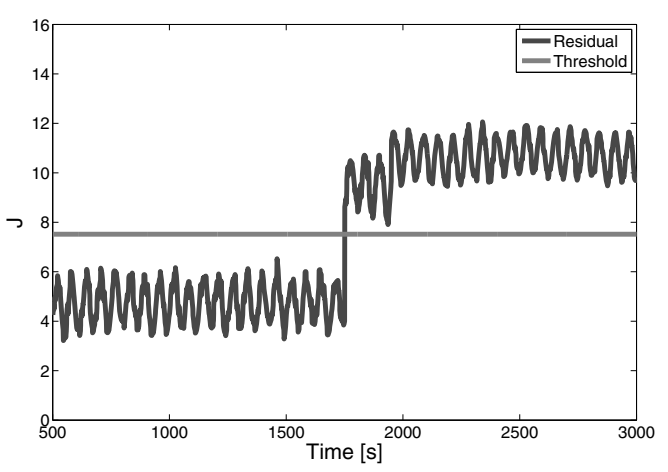

Fig. 1. Evaluated residual signal $J$.

norm based FAR and FDR. This problem is formulated as minimizing the FAR under a given FDR. The extension of the proposed approach to more general nonlinear systems will be the focus of our future work in this area.

\section{References}

Abbaszadeh, M. and Marquez, H. (2008). LMI optimization approach to robust $H_{\infty}$ filtering for discrete-time nonlinear uncertain systems, Proceedings of the American Control Conference, Washington, DC, USA, pp. 1905-1910.

Basseville, M. and Nikiforov, I.V. (1993). Detection of Abrupt Changes-Theory and Application, Prentice-Hall, Englewood Cliffs, NJ.

Blanke, M., Kinnaert, M., Lunze, J. and Staroswiecki, M. (2003). Diagnosis and Fault-Tolerant Control, Springer, New York, NY.

Chen, J. and Patton, R.J. (1999). Robust Model-Based Fault Diagnosis for Dynamic Systems, Kluwer Academic Publishers, Boston, MA.

Chen, W. and Saif, M. (2007). Observer-based strategies for actuator fault detection, isolation and estimation for certain class of uncertain nonlinear systems, IET Control Theory \& Applications 1(6): 1672-1680.

de Souza, C.E., Xie, L. and Wang, Y. (1993). $H_{\infty}$ filtering for a class of uncertain nonlinear systems, Systems and Control Letters 20(6): 419426.

Ding, S.X. (2008). Model-based Fault Diagnosis Techniques, Springer, Berlin.

Ding, S.X., Frank, P.M., Ding, E.L. and Jeinsch, T. (2000a). A unified approach to the optimization of fault detection systems, International Journal of Adaptive Control and Signal Processing 14(7): 725-745.

Ding, S.X., Frank, P.M., Ding, E.L. and Jeinsch, T. (2000b). Fault detection system design based on a new trade-off strategy, Proceedings of the 39th IEEE Conference on Decision and Control, Sydney, Australia, pp. 4144-4149.

Ding, S.X., Guo, L. and Frank, P.M. (1993). A frequency domain approach to fault detection of uncertain dynamic systems, Proceedings of the 32th IEEE Conference on Decision and Control, San Antonio, TX, USA, pp. 1722-1727.
Edelmayer, A., Bokor, J., Szab, Z. and Szigeti, F. (2004). Input reconstruction by means of system inversion: A geometric approach to fault detection and isolation in nonlinear systems, International Journal of Applied Mathematics and Computer Science 14(2): 189-199.

Ferrari, R., Parisini, T. and Polycarpou, M. (2007). A fault detection and isolation scheme for nonlinear uncertain discretetime systems, Proceedings of the 46th IEEE Conference on Decision and Control, New Orleans, LA, USA, pp. 1009_ 1014.

Frank, P.M. (1994). On-line fault detection in uncertain nonlinear systems using diagnostic observers: A survey, International Journal of Systems Science 25(12): 2129-2154.

Frank, P.M. and Ding, S.X. (1997). Survey of robust residual generation and evaluation methods in observer-based fault detection systems, Journal of Process Control 7(6): 403424.

Hammouri, H., Kinnaert, M. and Yaagoubi, E.H.E. (1999). Observer-based approach to fault detection and isolation for nonlinear systems, IEEE Transactions on Automatic Control 44(10): 1879-1884.

Henry, D. and Zolghadri, A. (2005). Design and analysis of robust residual generators for systems under feedback control, Automatica 41(2): 251-264.

Hou, M. and Patton, R.J. (1996). An LMI approach to $H_{-} / H_{\infty}$ fault detection observers, Proceedings of the UKACC International Conference on Control, Exeter, UK, pp. 1710-1715.

Khan, A.Q., Abid, M., Chen, W. and Ding, S.X. (2009). On optimal fault detection of nonlinear systems, Proceedings of the 48th IEEE Conference on Decision and Control, Shanghai, China, pp. 1032-1037.

Lai, T.L. and Shan, J.Z. (1999). Efficient recursive algorithms for detection of abrupt changes in signals and control systems, IEEE Transactions on Automatic Control 44(5): 952-966.

Narasimhan, S., Vachhani, P. and Rengaswamy, R. (2007). New nonlinear residual feedback observer for fault diagnosis in nonlinear systems, Automatica 44(9): 2222-2229.

Patton, R.J., Frank, P.M. and Clark, R.N. (2002). Issues of Fault Diagnosis for Dynamic Systems, Springer, Berlin.

Pertew, A.M., Marquez, H.J. and Zhao, Q. (2007). LMI-based sensor fault diagnosis for nonlinear Lipschitz systems, $\mathrm{Au}$ tomatica 43(8): 1464-1469.

Qiu, Z. and Gertler, J. (1993). Robust FDI systems and $H_{\infty}$ optimization, Proceedings of the American Control Conference, San Francisco, CA, USA, pp. 1710-1715.

Rajamani, R. (1998). Observers for Lipschitz nonlinear systems, IEEE Transactions on Automatic Control 43(3): 397-401.

Rajamani, R. and Ganguli, A. (2004). Sensor fault diagnostics for a class of non-linear systems using linear matrix inequalities, International Journal of Control 77(10): 920-930.

Shumsky, A. (2007). Redundancy relations for fault diagnosis in nonlinear uncertain systems, International Journal of Applied Mathematics and Computer Science 17(4): 477-489, DOI: 10.2478/v10006-007-0040-1. 
Wang, J.L., Yang, G. and Liu, J. (2007). An LMI approach to $H_{-}$index and mixed $H_{-} / H_{\infty}$ fault detection observer design, Automatica 43(9): 1656-1665.

Wang, Y., Xie, L. and Souza, C.E. (1992). Robust control of a class of uncertain nonlinear systems, Systems \& Control Letters 19(2): 139-149.

Xie, L., de Souza, C.E. and Wang, Y. (1996). Robust filtering for a class of discrete-time uncertain nonlinear systems: An $H_{\infty}$ approach, International Journal of Robust and Nonlinear Control 6(4): 297312.

Yaz, E. and Azemi, A. (1998). Actuator fault detection and isolation in nonlinear systems using LMIs and LMEs, Proceedings of the American Control Conference, Philadelphia, PA, USA, pp. 1590-1594.

Zhang, P. and Ding, S.X. (2008). An integrated trade-off design of observer based fault detection systems, Automatica 44(7): 1886-1894.

Zhang, P., Ding, S.X., Wang, G. and Zhou, D. (2005). Fault detection of linear discrete-time periodic systems, IEEE Transactions on Automatic Control 50(2): 239-244.

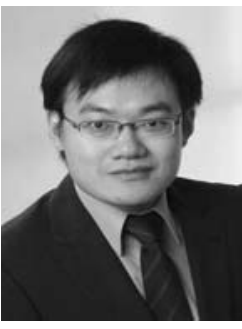

Wei Chen received the B.Sc. degree in automation from Xiamen University, China, in 2004, and the M.Sc. degree in control engineering from the China Academy of Space Technology, Beijing, in 2007. Since 2007, he has been with the Institute of Automatic Control and Complex Systems, University of Duisburg-Essen, Germany, where he is pursuing his $\mathrm{Ph} . \mathrm{D}$. in electrical engineering. His research interests include fault diagnosis for nonlinear systems, nonlinear observer design and energy-based system analysis.

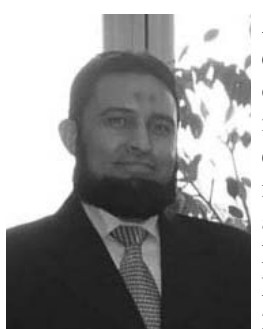

Abdul Q. Khan received the B.Sc. degree in electrical engineering from the NWFP University of Engineering and Technology, Peshawar, Pakistan, in 2002, and the M.Sc. degree in systems engineering from the Pakistan Institute of Engineering and Applied Sciences (PIEAS), Islamabad, in 2004. During the period of 2004-2006, he was a lecturer in the Department of Electrical Engineering, PIEAS. During the period of 20072010, he was a guest scientist in the Institute of Automatic Control and Complex Systems, University of DuisburgEssen, Germany. He received his Ph.D. degree in electrical engineering from the University of Duisburg-Essen in 2010. Currently he is an assistant professor in the PIEAS. His research interest include fault diagnosis in technical processes, linear and nonlinear observer design, robust control of nonlinear systems, and LMI based optimal design.

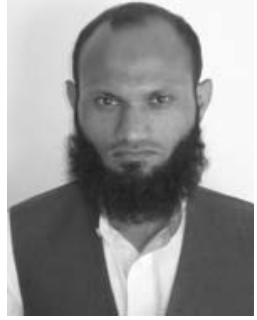

Muhammad Abid received the M.Sc. degree (systems engineering) from the Pakistan Institute of Engineering and Applied Sciences (PIEAS), Islamabad, in 2004, and the Ph.D. degree from the Institute of Automatic Control and Complex Systems, University of Duisburg-Essen, Germany, in 2010. Currently, he is an assistant professor in the PIEAS. His research interests include model-based fault detection in nonlinear systems, and robust and optimal control.

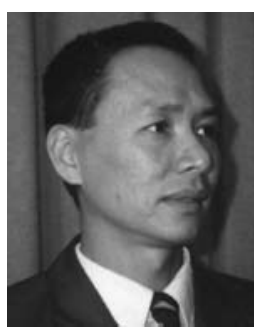

Steven X. Ding received the Ph.D. degree in electrical engineering from the Gerhard Mercator University of Duisburg, Germany, in 1992. From 1992 to 1994, he was an R\&D engineer at Rheinmetall GmbH. From 1995 to 2001, he was a professor of control engineering at the Lausitz University of Applied Sciences in Senftenberg, Germany, and served as a vice president of this university in the years 1998-2000. He is currently a professor of control engineering and the head of the Institute for Automatic Control and Complex Systems (AKS) at the University of Duisburg-Essen, Germany. His research interests are model-based and data-driven fault diagnosis, fault tolerant systems and their application in industry with a focus on automotive systems and chemical processes.

Received: 28 February 2010

Revised: 15 November 2010 